University of Nebraska - Lincoln

DigitalCommons@University of Nebraska - Lincoln

$11-8-2001$

\title{
Monte Carlo simulation of homogeneous binary vapor-liquid nucleation: Mutual enhancement of nucleation in a partially miscible system
}

S. Yoo

University of Nebraska-Lincoln

K.J. Oh

University of Nebraska-Lincoln

Xiao Cheng Zeng

University of Nebraska-Lincoln, xzeng1@unl.edu

Follow this and additional works at: https://digitalcommons.unl.edu/chemzeng

Part of the Chemistry Commons

Yoo, S.; Oh, K.J.; and Zeng, Xiao Cheng, "Monte Carlo simulation of homogeneous binary vapor-liquid nucleation: Mutual enhancement of nucleation in a partially miscible system" (2001). Xiao Cheng Zeng Publications. 42.

https://digitalcommons.unl.edu/chemzeng/42

This Article is brought to you for free and open access by the Published Research - Department of Chemistry at DigitalCommons@University of Nebraska - Lincoln. It has been accepted for inclusion in Xiao Cheng Zeng Publications by an authorized administrator of DigitalCommons@University of Nebraska - Lincoln. 


\title{
Monte Carlo simulation of homogeneous binary vapor-liquid nucleation: Mutual enhancement of nucleation in a partially miscible system
}

\author{
S. Yoo, K. J. Oh, and X. C. Zeng \\ Department of Chemistry, University of Nebraska-Lincoln, Lincoln, Nebraska 68588
}

(Received 27 June 2001; accepted 15 August 2001)

\begin{abstract}
By means of a constrained canonical-ensemble Monte Carlo technique the equilibrium physical cluster distribution $\left\{n_{i j}\right\}$ in a supersaturated vapor mixture is computed. From this the intensive Gibbs free energy of $i j$-cluster formation $\Delta G_{i j}$ is obtained via the relation $n_{i j}=N e^{-\Delta G_{i j} / k_{B} T}$, where $N$ is the number of molecules in the system, $T$ is temperature, and $k_{B}$ is the Boltzmann constant. The saddle point on the free energy surface $\Delta G_{i j}$ versus $i$ and $j$ provides a measure of the activation barrier to nucleation $\Delta G^{*}$. A statistical mechanical formula of $\Delta G_{i j}$ is derived based on the physical-cluster theory of nucleation, and from which a molecular interpretation of $\Delta G_{i j}$ is given. We applied the Monte Carlo method to investigate the mutual enhancement of nucleation in a binary model system which is composed of spherical Lennard-Jones monomers and rigid Lennard-Jones dimers consisting of a monophilic atom and a monophobic atom. This model system bears some qualitative similarity to the partially miscible water/higher alcohol systems in which the mutual enhancement of nucleation has been observed experimentally. The simulation confirms the existence of mutual enhancement of nucleation in monomer-rich vapors, as originally predicted by Napari and Laaksonen from a density-functional theory [Napari and Laaksonen, Phys. Rev. Lett. 84, 2184 (2000)]. The nucleation enhancement is also seen in dimer-rich vapors at high supersaturations.

(C) 2001 American Institute of Physics. [DOI: 10.1063/1.1409364]
\end{abstract}

\section{INTRODUCTION}

A better understanding of binary vapor-liquid nucleation is of importance in many areas of science and technology, particularly in atmospheric science. ${ }^{1}$ The classical theory of binary nucleation developed by Reiss ${ }^{2}$ can be traced back to the middle of the past century. This theory has been applied to sulfuric acid/water system by Doyle. ${ }^{3}$ Wilemski ${ }^{4,5}$ later proposed a thermodynamically consistent classical theory, known as the "revised classical theory of nucleation." Although the revised theory works rather well for nearly ideal binary systems, a number of recent experimental measurements ${ }^{6-8}$ indicate that the theory can yield unphysical results for strongly nonideal mixtures such as water/alcohol. This deficiency of the classical theory has prompted the development of statistical mechanics based theory of nucleation, for example, the density functional theory, ${ }^{9-12}$ the transition-state theory, ${ }^{13}$ as well as various molecular based computer simulation methodology. ${ }^{14-25}$ The latter is particularly useful to obtain quantitative information on the equilibrium physical cluster distribution and the activation barrier to nucleation. For a unary system the intensity free energy of formation $\Delta G_{i}$ of $i$-clusters is given by

$$
\Delta G_{i}=-k_{B} T \ln n_{i} / N,
$$

where $n_{i}$ is the equilibrium number of $i$-clusters, $T$ is temperature, $k_{B}$ is the Boltzmann constant, and $N$ is the number of molecules of the system. Statistical mechanics based formulas for $\Delta G_{i}$ have been derived recently. ${ }^{14,15,20,24,26}$ A molecular interpretation of $\Delta G_{i}$ is given by Reiss and Bowles, ${ }^{26}$ that is, $\Delta G_{i}$ can be viewed as the free energy change associated with the gathering of $i$ molecules located in the vol- ume $V$ of the system into the volume per molecule, $v$ $=V / N$, and forming them into an $i$-cluster there.

In contrast to unary systems, fewer computational studies have been devoted to vapor-liquid nucleation in binary systems. $^{27-29}$ For the latter, Eq. (1) becomes ${ }^{5,30}$

$$
\Delta G_{i j}=-k_{B} T \ln n_{i j} / N,
$$

where $\Delta G_{i j}$ is the "intensive" Gibbs free energy of formation of $i j$-clusters containing $i$ molecules of species 1 and $j$ molecules of species $2, n_{i j}$ is the equilibrium number of $i j$ clusters. To our knowledge, the statistical mechanical formula of $\Delta G_{i j}$ based on the physical-cluster theory (as that of $\Delta G_{i}$ for unary systems ${ }^{14,26}$ ) has not yet been derived. In this paper, we first extend the physical-cluster theory for unary systems $^{24}$ to the binary systems and to obtain a statistical mechanical expression of $\Delta G_{i j}$. We then investigate an unusual nucleation behavior-the mutual enhancement of nucleation in certain binary systems. Strey and co-workers ${ }^{31}$ have recently observed that in binary vapor mixtures of water and higher alcohols the vapor-to-liquid nucleation is mutually enhanced relative to an "ideal" binary mixture. This behavior was somewhat unexpected in the sense that higher alcohols do not mix well with water in the liquid state (i.e., water and higher alcohol solutions are partially miscible) and that most previous theoretical ${ }^{12,32,33}$ and computational $^{29}$ studies indicated that if a simple fluid mixture exhibits partial miscibility in the liquid state, "mutual hindrance of nucleation" is most likely to occur in the vapor-to-liquid nucleation. Clearly, simple fluid models cannot account for the apparent mutual enhancement of nucleation behavior in water/alcohol systems. Recently Napari and Laaksonen ${ }^{34}$ de- 
vised a model system to mimic binary nucleation in water/ alcohol systems. This model system consists of monomers (as water) and dimer molecules (as higher alcohols). The dimer molecule consists of a monophilic atom (as the hydroxy group of alcohol) and a monophobic atom (as the hydrocarbon group of alcohol). From a density-functional theory of nucleation they found evidence of mutual enhancement of nucleation in a monomer-rich vapor, but not in the dimer-rich vapor. In this paper, we show that the Monte Carlo simulation supports Napari and Laaksonen's conclusion in that the mutual enhancement of nucleation indeed occurs in the monomer-rich vapor. Moreover, we will also show evidence that mutual enhancement of nucleation can occur in highly supersaturated dimer-rich vapors.

In Sec. II, we derive a statistical mechanical expression of chemical potential for the ij-clusters in a supersaturated binary vapor (Sec. II A). We then use this chemical potential to obtain the intensive Gibbs free energy formation of $i j$ clusters, $\Delta G_{i j}$ (See. II B). A molecular interpretation of $\Delta G_{i j}$ is also given. In Sec. III, we describe the model system and the Monte Carlo method. The results and discussions are presented in Sec. IV. The conclusion is summarized in Sec. V. Finally, a derivation of the law of mass action is given in the Appendix.

\section{STATISTICAL MECHANICAL EXPRESSION OF $\Delta G_{i j}$}

\section{A. Chemical potential of $i j$-clusters}

As discussed in a previous work for unary systems, ${ }^{24}$ we consider a supersaturated vapor as a polyatomic gas. The interaction between clusters and gas-phase molecules is assumed to be negligible. Clusters containing the same number of molecules and with the same composition are indistinguishable and are considered to have the same averaged (excluded) volume. Here, we denote A molecules and B molecules as species 1 and 2, respectively. For a supersaturated vapor at a given volume $V$, temperature $T$, mole fraction $x_{\mathrm{A}}$, and at the constrained equilibrium (the constraint is the maximum size of the cluster), the partition function of an $i j$-cluster can be written as

$$
\begin{aligned}
q_{i j}= & \frac{1}{i ! j ! \Lambda_{\mathrm{A}}^{3 i} \Lambda_{\mathrm{B}}^{3 j}} \int_{V} \exp \left(-U_{i j} / k_{B} T\right) d \boldsymbol{r}_{\mathrm{A} 1} \cdots d \boldsymbol{r}_{\mathrm{A} i} d \boldsymbol{r}_{\mathrm{B} 1} \cdots d \boldsymbol{r}_{\mathrm{B} j} \\
= & \frac{\left(i+m_{\mathrm{B} j} j m_{\mathrm{A}}\right)^{3}}{i ! j ! \Lambda_{\mathrm{A}}^{3 i} \Lambda_{\mathrm{B}}^{3 j}} \int_{V} d \boldsymbol{R} \\
& \times \int_{c} \exp \left(-U_{i j} / k_{B} T\right) d \boldsymbol{r}^{\prime}{ }_{\mathrm{A} 1} \cdots d \boldsymbol{r}^{\prime}{ }_{\mathrm{A} i-1} d \boldsymbol{r}^{\prime}{ }_{\mathrm{B} 1} \cdots d \boldsymbol{r}^{\prime}{ }_{\mathrm{B} j} \\
= & \frac{V}{\Lambda_{i j}^{3}} \frac{\left(i+m_{\mathrm{B}} j / m_{\mathrm{A}}\right)^{3} \Lambda_{i j}^{3}}{i ! j ! \Lambda_{\mathrm{A}}^{3 i} \Lambda_{\mathrm{B}}^{3 j}} \\
& \times \int_{c} \exp \left(-U_{i j} / k_{B} T\right) d \boldsymbol{r}^{\prime}{ }_{\mathrm{A} 1} \cdots d \boldsymbol{r}^{\prime}{ }_{\mathrm{A} i-1} d \boldsymbol{r}^{\prime}{ }_{\mathrm{B} 1} \cdots d \boldsymbol{r}^{\prime}{ }_{\mathrm{B} j},
\end{aligned}
$$

where $U_{i j}$ is the internal energy of the $i j$-cluster, $\boldsymbol{r}_{\alpha}$ is the positional coordinate of the molecule of species $\alpha$, the primed coordinates are measured from the center-of-mass
(COM) coordinate of the $i j$-cluster, and $R$ is the COM coordinate. In Eq. (3), the factor $\left(i+m_{\mathrm{B}} j / m_{\mathrm{A}}\right)^{3}$ results from the Jacobian of the coordinate transformation of one A molecule to the COM coordinate, and $m_{\alpha}$ is the mass of the molecule of species $\alpha$; the subscript $c$ refers to the cluster criterion, ${ }^{25}$ $\Lambda_{\alpha}$ is the thermal de Broglie wavelength of molecules of species $\alpha$,

$$
\Lambda_{\alpha}=\left[\frac{h^{2}}{2 \pi m_{\alpha} k_{B} T}\right]^{1 / 2},
$$

where $h$ is Planck's constant, and that of $i j$-clusters is given by

$$
\Lambda_{i j}=\left[\frac{h^{2}}{2 \pi\left(i m_{\mathrm{A}}+j m_{\mathrm{B}}\right) k_{B} T}\right]^{1 / 2} .
$$

Denoting the most probable cluster distribution as $\left\{n_{i j}\right\}$, the total number of clusters in the system is given by $N$ $=\Sigma_{i j} n_{i j}$. Since every $i j$-cluster possesses an excluded volume, the real volume of the system for clusters to translate, $V^{e}$, is slightly less than the total volume of the system $V$, that is,

$$
V^{e}=V-\sum_{i j} v_{i j} n_{i j},
$$

where $v_{i j}$ is the averaged excluded volume of an $i j$-cluster. As a consequence, the integration over the COM coordinate $R$ yields $V^{e}$ rather than $V$. The replacement of $V$ by $V^{e}$ in Eq. (3) gives

$$
\begin{aligned}
q_{i j}= & \frac{V^{e}}{\Lambda_{i j}^{3}} \frac{\left(i+m_{\mathrm{B}} j / m_{\mathrm{A}}\right)^{3} \Lambda_{i j}^{3}}{i ! j ! \Lambda_{\mathrm{A}}^{3 i} \Lambda_{\mathrm{B}}^{3 j}} \\
& \times \int_{c} \exp \left(-U_{i j} / k_{B} T\right) d \boldsymbol{r}_{\mathrm{A} 1}^{\prime} \cdots d \boldsymbol{r}^{\prime}{ }_{\mathrm{A} i-1} d \boldsymbol{r}^{\prime}{ }_{\mathrm{B} 1} \cdots d \boldsymbol{r}^{\prime}{ }_{\mathrm{B} j} \\
= & q_{i j}^{t} q_{i j}^{*},
\end{aligned}
$$

where $q_{i j}^{t}$ is the translational partition function of an $i j$ cluster, that is

$$
q_{i j}^{t}=\frac{V^{e}}{\Lambda_{i j}^{3}},
$$

and $q_{i j}^{*}$ is the partition function of the $i j$-cluster in the COM coordinate, that is

$$
\begin{aligned}
q_{i j}^{*}= & \frac{\left(i+m_{\mathrm{B}} j / m_{\mathrm{A}}\right)^{3} \Lambda_{i j}^{3}}{i ! j ! \Lambda_{\mathrm{A}}^{3 i} \Lambda_{\mathrm{B}}^{3 j}} \\
& \times \int_{c} \exp \left(-U_{i j} / k_{B} T\right) d \boldsymbol{r}^{\prime}{ }_{\mathrm{A} 1} \cdots d \boldsymbol{r}^{\prime}{ }_{\mathrm{A} i-1} d \boldsymbol{r}^{\prime}{ }_{\mathrm{B} 1} \cdots d \boldsymbol{r}^{\prime}{ }_{\mathrm{B} j} .
\end{aligned}
$$

The internal Helmholtz free energy of the $i j$-cluster is then given by

$$
f_{i j}^{*}=-k_{B} T \ln q_{i j}^{*} .
$$

Assuming all the $n_{i j}$ number of $i j$-clusters are indistinguishable, the canonical partition function of the entire system can then be written in terms of $q_{i j}$, that is, 


$$
Q=\prod_{i j} \frac{q_{i j}^{n_{i j}}}{n_{i j} !} .
$$

Thus, the Helmholtz free energy $A$ of the system can be obtained via the equation

$$
A=-k_{B} T \ln Q=-k_{B} T \sum_{i j}\left\{n_{i j} \ln q_{i j}-n_{i j} \ln n_{i j}+n_{i j}\right\},
$$

where the Stirling's formula is used for computing $n_{i j} !$. From the standard definition of the chemicala potential of $i j$-clusters, we have

$$
\begin{aligned}
\mu_{i j} & =\left[\frac{\partial A}{\partial n_{i j}}\right]_{V, T, n_{k l \neq i j}} \\
& =-k_{b} T\left[\ln \frac{q_{i j}}{n_{i j}}-\frac{v_{i j}}{V^{e}} \sum_{i j} n_{i j}\right] \\
& =-k_{B} T \ln \frac{q_{i j}^{t}}{n_{i j}}-k_{B} T \ln q_{i j}^{*}+p v_{i j} \\
& =-k_{B} T \ln \frac{q_{i j}^{t}}{n_{i j}}+f_{i j}^{*}+p v_{i j} .
\end{aligned}
$$

In deriving Eq. (11), we used the relation $\partial V^{e} / \partial n_{i j}=v_{i j}$, and $p \approx N k_{B} T / V^{e}$ (assuming the supersaturated vapor behaves like an ideal gas). Substitution of Eq. (6) into Eq. (11) yields

$$
\begin{aligned}
\mu_{i j} & =-k_{B} T \ln \frac{V^{e}}{\Lambda_{i j}^{3} n_{i j}}+f_{i j}^{*}+p v_{i j}, \\
& =-k_{B} T \ln \frac{k_{B} T}{\Lambda_{i j}^{3} p_{i j}}+f_{i j}^{*}+p v_{i j},
\end{aligned}
$$

where $p_{i j}=n_{i j} k_{B} T / V \approx n_{i j} k_{B} T / V^{e}$ is the partial pressure of $i j$-clusters.

\section{B. Free energy of cluster formation $\Delta G_{i j}$}

For unary systems, ${ }^{25}$ the free energy of cluster formation $\Delta G_{i}$ can be defined as the chemical potential change associated with transforming $i$ monomers into an $i$-cluster at constant pressure of the supersaturated vapor $p$. With this definition, we obtained a statistical mechanical expression of $\Delta G_{i}$ identical to that of Reiss and Bowles. ${ }^{26}$ To extend this definition to binary systems, we consider the following gasphase chemical reaction under the condition of fixed pressure $p$, temperature $T$, and mole fraction $x_{\mathrm{A}}$,

$$
i \mathrm{~A}+j \mathrm{~B} \rightleftharpoons \mathrm{A}_{i} \mathrm{~B}_{j},
$$

where species 1 and 2 are denoted by $\mathrm{A}$ and $\mathrm{B}$, and $\mathrm{A}_{i} \mathrm{~B}_{j}$ denotes the $i j$-clusters. Therefore, the Gibbs free energy of cluster formation $\Delta G_{i j}$ at equilibrium can be given by

$$
\Delta G_{i j}(p)=\mu_{i j}(p)-i \mu_{\mathrm{A}}\left(p_{\mathrm{A}}\right)-j \mu_{\mathrm{B}}\left(p_{\mathrm{B}}\right) .
$$

This definition is similar to that for unary systems. But note that the chemical potential of each molecular species has $p_{\alpha}$, the partial pressure of that species, as an independent variable. Hence, a molecular interpretation can be that $\Delta G_{i j}$ is the chemical potential change associated with the gathering of $i$ monomers of A molecules from the system at pressure
$p_{\mathrm{A}}$ and $j$ monomers of $\mathrm{B}$ molecules from the system at pressure $p_{\mathrm{B}}$ and forming an $i j$-cluster at the pressure $p$. Moreover, with the law of mass action (see Appendix), $\mu_{i j}\left(p_{i j}\right)$ $=i \mu_{\mathrm{A}}\left(p_{\mathrm{A}}\right)+j \mu_{\mathrm{B}}\left(p_{\mathrm{B}}\right), \Delta G_{i j}$ can be rewritten as the chemical potential difference of the $i j$-cluster at its partial pressure $p_{i j}$ and $p$, that is

$$
\Delta G_{i j}(p)=\mu_{i j}(p)-\mu_{i j}\left(p_{i j}\right) .
$$

Furthermore, for dilute gases, it is reasonable to express the term $\mu_{i j}(p)$ in both Eqs. (14) and (15) with an ideal gas formula,

$$
\mu_{i j}(p)=\mu_{i j}\left(p_{i j}\right)+k_{B} T \ln \frac{p}{p_{i j}} \approx \mu_{i j}\left(p_{i j}\right)-k_{B} T \ln \frac{n_{i j}}{N} .
$$

Substituting Eq. (16) into Eq. (15) yields Eq. (2).

\section{THE SYSTEM AND MONTE CARLO SIMULATION}

To study the mutual enhancement of nucleation in binary mixtures, we employed the Napari-Laaksonnen (NL) model system which consists of spherical atoms (monomers) and diatomic dumbbell molecules (dimers). The interatomic interactions are described by the Lennard-Jones potential,

$u(r)=4 \epsilon_{i j}\left[\left(\sigma_{i j} / r\right)^{12}-\left(\sigma_{i j} / r\right)^{6}\right] \quad(i, j=0,1,2)$,

where $r$ is the interatomic distance, $\sigma_{i j}$ and $\epsilon_{i j}$ are the Lennard-Jones size and energy parameter, respectively. Here, the index 0 refers to the monomer whereas indices 1 and 2 refer to the monophilic and monophobic atom in the dimer, respectively. The diameters of all atoms are the same, i.e., $\sigma_{i i}=\sigma(i=0,1,2)$. The bond length of the dimer molecule is fixed to $\sigma$. The energy parameters between unlike atoms are redefined with an additional parameter $k_{i j}$, i.e.,

$$
\epsilon_{i j}=\left(1-k_{i j}\right) \sqrt{\epsilon_{i i} \epsilon_{j j}} \quad(i \neq j) .
$$

Thus, the interaction between unlike atoms can be adjusted by changing the value of $k_{i j}$.

The Monte Carlo (MC) simulation is carried out in the canonical ensemble with periodic boundary conditions. Since the dimer is a linear rigid molecule, rotation steps are involved in the MC trial moves, in which a dimer is randomly selected and then rotated at a random angle with respect to an arbitrary space-fixed coordinate. ${ }^{35}$ After each MC translational or rotational move, all clusters in the system are identified according to Stillinger's criterion, ${ }^{36}$ that is, any atom in a cluster is within a cutoff distance $r_{\mathrm{cl}}$ to, at least, another atom in that cluster. Following previous studies, ${ }^{16,17,20,24,25}$ $r_{\mathrm{cl}}$ is set to $1.5 \sigma$. The maximal cluster size is set at $i_{\max }$. Here $i_{\max }$ acts as a constraint to maintain the supersaturated vapor in the constrained equilibrium. ${ }^{24}$ If a MC move results in a cluster with size greater than $i_{\max }$, that MC move is rejected.

The cluster distribution $\left\{n_{i j}\right\}$ at the (constrained) equilibrium can be computed by counting all $i j$-clusters (containing $i$ monomers and $j$ dimers) at each MC cycle and by averaging these countings over a sufficiently large number of MC cycles. Once $\left\{n_{i j}\right\}$ is known, the intensive Gibbs free energy of formation can be determined via the equation, 


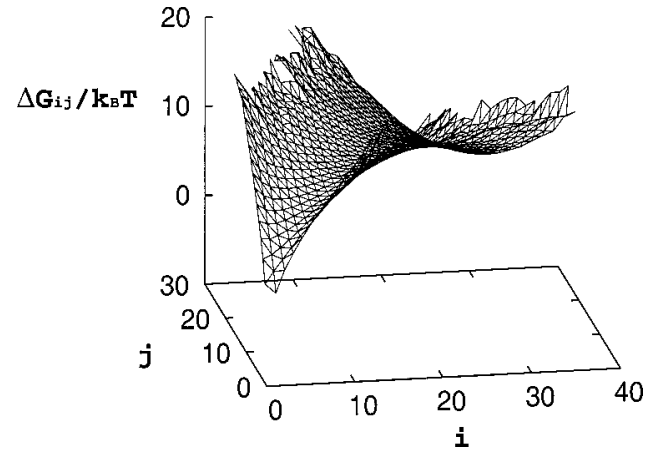

FIG. 1. The free-energy surface $\Delta G_{i j}$ for the NL system at $x_{m}=0.6$ vs the monomer number $i$ and dimer number $j$ in $i j$-clusters.

$$
\Delta G_{i j} / k_{B} T=-\ln \left(\frac{n_{i j}}{N_{1}}\right),
$$

where, $N_{1}=n_{10}+n_{01} \approx N$. In all simulations, we set the number of molecules in the system 4000, and temperature $T$ $=0.7 \epsilon / k_{B}$ with $i_{\max }=46$. The Lennard-Jones potential is truncated at $r_{c}=3.5 \sigma$ and shifted such that the potential is zero at $r_{c}$.

\section{RESULT AND DISCUSSION}

In order to compare the MC simulation results with those of density-functional theory, we used the same notations as the previous publication. ${ }^{34}$ In particular, the energy parameters for the partially miscible system are $\epsilon=\epsilon_{00}$ $=1.0, \epsilon_{11} / \epsilon_{00}=\epsilon_{22} / \epsilon_{00}=0.6, k_{01}=-0.7, k_{02}=0.8$, and $k_{12}$ $=0.0$ (hereafter this system is called the NL system). For the reference "ideal" binary mixture system, ${ }^{12,29}$ we set the energy parameters for unlike atoms to be $\epsilon_{01}=\left(\epsilon_{00}+\epsilon_{11}\right) / 2$ and $\epsilon_{02}=\left(\epsilon_{00}+\epsilon_{22}\right) / 2$. Thus, since the attractive interactions between the monomer and both atoms of the dimer are the same the reference system is expected to exhibit "ideal" mixing behavior. ${ }^{12}$

Figure 1 shows the free energy of formation surface $\Delta G_{i j}$, spanned by the monomer number $i$ and dimer number $j$ in an $i j$-cluster, for the NL system with the mole fraction of monomers $x_{m}=0.6$. A saddle point on the surface can be clearly seen, at which the free energy is denoted by $\Delta G^{*}$. The value of $\Delta G^{*}$ provides a measure of the activationbarrier height to binary nucleation because the nucleation flux is expected to most likely traverse through the valley

TABLE I. Numerical results of NL systems at temperature $T=0.7 \epsilon / k_{B}$, total molecule number 4000 , and volume $V=200000 \sigma^{3}$.

\begin{tabular}{cccc}
\hline \hline$x_{m}{ }^{\mathrm{a}}$ & $\Delta G^{*} / k_{B} T$ & $\left(i^{*}, j^{*}\right)^{\mathrm{b}}$ & $p^{\mathrm{c}}$ \\
\hline 0.1 & 7.82 & $(0,31)$ & 0.006787 \\
0.2 & 9.74 & $(5,24)$ & 0.006976 \\
0.4 & 10.82 & $(11,16)$ & 0.007116 \\
0.6 & 11.20 & $(14,12)$ & 0.007519 \\
0.8 & 11.73 & $(19,7)$ & 0.008471 \\
1.0 & 11.56 & $(33,0)$ & 0.010273 \\
\hline \hline
\end{tabular}

${ }^{a} x_{m}$ is the mole fraction of monomer.

${ }^{\mathrm{b}} i^{*}$ and $j^{*}$ are the number of monomers and dimers in the critical cluster, respectively.

${ }^{c} p=N k_{B} T / V$, where $N$ is the total number of clusters. $p$ is in units of $\epsilon / \sigma^{3}$.
TABLE II. Numerical results of reference system at temperature $T$ $=0.7 \epsilon / k_{B}$, total molecule number 4000 .

\begin{tabular}{ccccc}
\hline \hline$x_{m}$ & $\Delta G^{*} / k_{B} T$ & $\left(i^{*}, j^{*}\right)$ & $p$ & $V^{\mathrm{a}}$ \\
\hline 0.0 & 7.82 & $(0,31)$ & 0.006787 & 200000 \\
0.2 & 10.88 & $(7,30)$ & 0.006954 & 245000 \\
0.4 & 12.87 & $(13,25)$ & 0.007128 & 270000 \\
0.6 & 14.07 & $(20,16)$ & 0.007537 & 270000 \\
0.8 & 13.61 & $(22,9)$ & 0.008459 & 245000 \\
1.0 & 11.56 & $(33,0)$ & 0.010273 & 200000 \\
\hline \hline
\end{tabular}

${ }^{\mathrm{a}} V$ is in units of $\sigma^{3}$.

shown in Fig. 1, where the highest point is the saddle. Note that $\Delta G^{*}$ is also the free energy of formation of the critical cluster containing $i^{*}$ number of monomers and $j^{*}$ of dimer molecules.

The simulation results for the NL system at various $x_{m}$ are shown in Table I while those for the reference system are shown in Table II. In particular, $\Delta G^{*}$ for both systems is plotted in Fig. 2. Since $\Delta G^{*}$ not only depends on $x_{m}$ but also on the total vapor pressure $p$, or, equivalently, on the activities (as used by experimentalists) $a_{\alpha}=p_{\alpha} / p_{\alpha}^{0}$, where $p_{\alpha}^{0}$ is the equilibrium vapor pressure over pure liquid $\alpha$ and $p_{\alpha}$ is the partial vapor pressure of the $\alpha$ species, $\Delta G^{*}$ of the two systems should be compared with each other at the same given $p$ and $x_{m}$. To this end, we varied the volume of the reference system until its vapor pressure is about equal to that of the NL system. Here, the vapor pressure is evaluated approximately using the ideal gas relationship $p=N k_{B} T / V$, where $N$ is the averaged number of clusters. As shown in Table I the difference in the vapor pressure between the NL and the reference system is less than $0.3 \%$.

Figure 2 provides an evidence of the mutual enhancement of nucleation in the partially miscible NL system in comparison with the reference system. For the former, not only the mutual enhancement of nucleation occurs in the monomer-rich vapor $\left(x_{m}=0.8\right)$ but also in the dimer-rich vapor $\left(x_{m}=0.2\right)$. In their theoretical work, however, Napari and Laaksonen found that the mutual enhancement of nucleation occurred only in monomer-rich vapors. ${ }^{34}$ They attributed the mutual enhancement of nucleation to the reduction of the surface tension of the critical cluster. This reduction

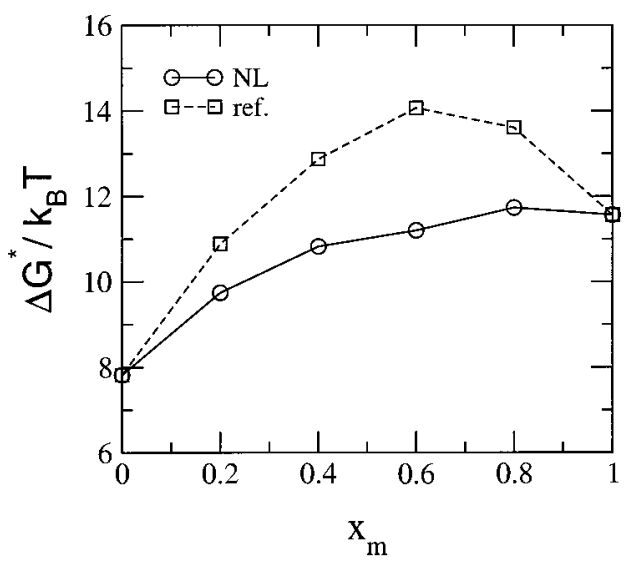

FIG. 2. Comparison of the free energy of formation of critical cluster $\Delta G^{*} / k_{B} T$ of the NL system to that of the reference system. Both systems are given the same vapor pressure $p$ and the monomer mole fraction $x_{m}$. 
(a)

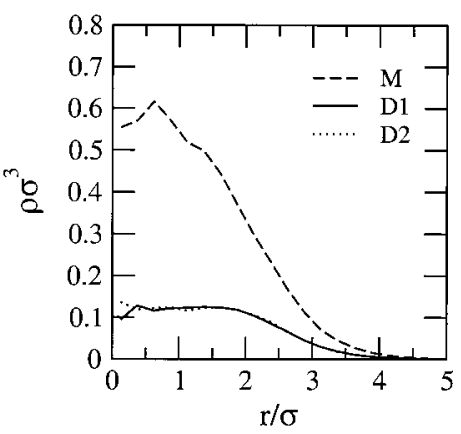

(b)

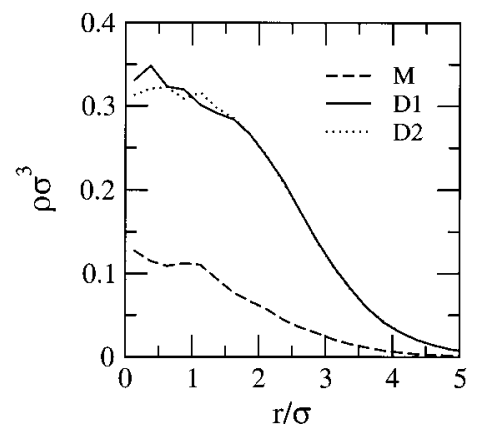

FIG. 3. The atomic density profiles of the monomer $(M)$, monophilic atom of the dimer (D1), and monophobic atom of the dimer (D2) for the reference system of (a) the monomer-rich vapor, $x_{m}=0.8$ and (b) the dimer-rich vapor, $x_{m}=0.2$.

stems from the appearance of orientational surface order for the dimers at the surface of clusters. To verify this surface order, we calculated the atomic density profiles of the monophilic and monophobic atom of the dimer, respectively, which are defined as the average number of atoms per unit volume at a distance $r$ from the COM of the cluster. Results are shown in Figs. 3 and 4 for the reference and NL system, respectively. In both cases, the size of clusters used in the averaging is 46 (slightly larger than the critical cluster) for the purpose of the getting good statistics since this cluster is the most numerous postcritical cluster in the constrained equilibrium distribution. Note that the size of the critical cluster $\left(i^{*}+j^{*}\right)$ ranges from 26 to 29 for the NL system (Table I) and from 31 to 38 for the reference system (Table II). Figure 5 shows the normalized probability distribution versus the mode fraction of monomers $x_{m}^{c}$ in those clusters containing 46 molecules for both systems and at both monomer-rich and dimer-rich mole fractions. It can be seen that the mole fraction corresponding to the maximal probability is quite close to $x_{m}$.

For the reference system, as shown in Fig. 3, the atomic density profiles of the dimer nearly overlap with each other, indicating nonexistence of the orientational surface order. In contrast, the orientational surface order is observed in the NL system as shown in Fig. 4, at both the monomer-rich [Fig. 4(a)] and dimer-rich vapor [Fig. 4(b)] mole fractions. Indeed, Fig. 4(a) resembles the corresponding density profile shown in Ref. 33. The fact that the orientational surface order of dimers is also seen in the dimer-rich vapor confirms the $\Delta G^{*}$ results. Naturally, a question is why Napari and Laaksonen did not see this enhancement in the dimer-rich vapor. (a)

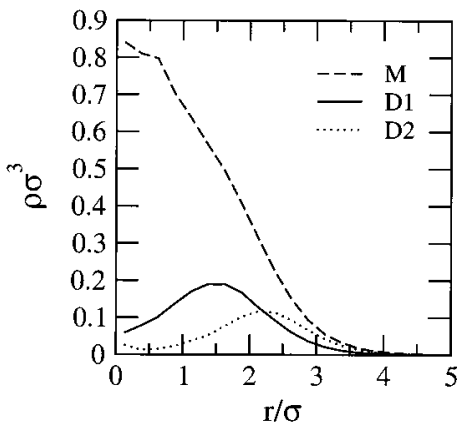

(b)

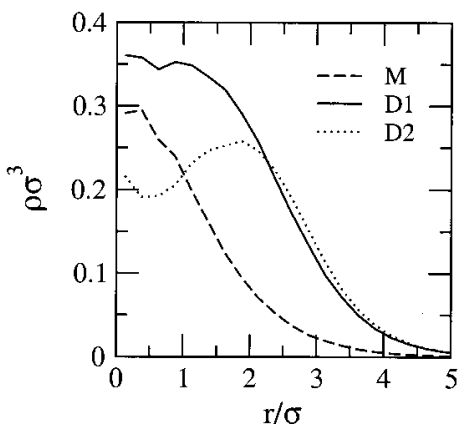

FIG. 4. The atomic density profiles of the monomer (M), monophilic atom of the dimer (D1), and monophobic atom of the dimer (D2) for the NL system of (a) the monomer-rich vapor, $x_{m}=0.8$ and (b) the diatomicmolecule-rich vapor, $x_{m}=0.2$.

A more careful inspection of the atomic density profiles in the dimer-rich vapor indicates that the supersaturation chosen in Napari-Laaksonen study is much lower than in ours. In fact, in the simulation the binary vapor is highly supersaturated. As a consequence, the activation barrier to nucleation is much lower here and so is the size of the critical clusters. However, the mole fraction of monomers, $x_{m}^{c *}$ $=i^{*} /\left(i^{*}+j^{*}\right)$, is higher for the simulation. As the size of the critical clusters decreases, it appears that the interfacial interaction between the monomers in the inner-core region and dimers on the surface of the cluster becomes more and more significant. Such an interfacial interaction is mainly responsible for the orientational surface order of dimers on the surface of the cluster due to the uneven interaction between the monomers and the monophilic and monophobic atoms of the dimers. On the other hand, for a large critical droplet with a very low mole fraction of monomers (as in the case of Ref. 33), the "bulk" characteristics of the dimer liquid (or volume effect of dimers as opposed to the interfacial effect between the monomers and dimers) is dominant. Therefore, the two atomic density profiles of the dimers nearly overlap with each other without showing any sign of orientational surface order of dimers.

\section{CONCLUSION}

We have extended a previously developed physicalcluster theory of nucleation for unary systems to binary systems. As a result, a molecular interpretation of the intensive Gibbs free energy of formation for $i j$-clusters is given, that is, $\Delta G_{i j}$ is the chemical potential change associated with gathering $i$ monomers of $A$ molecules from the system at pressure 
(a)

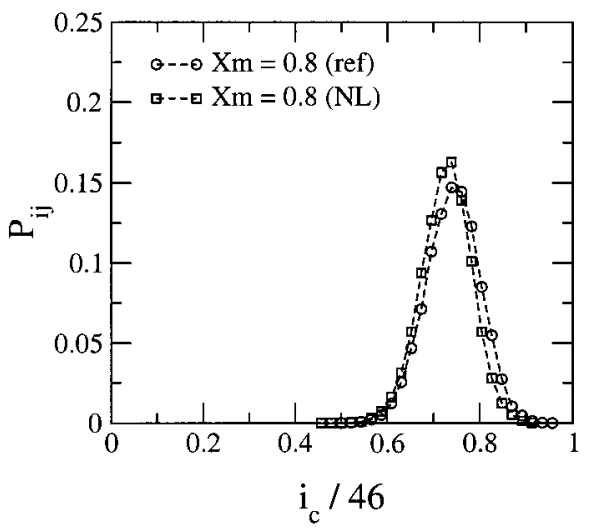

(b)

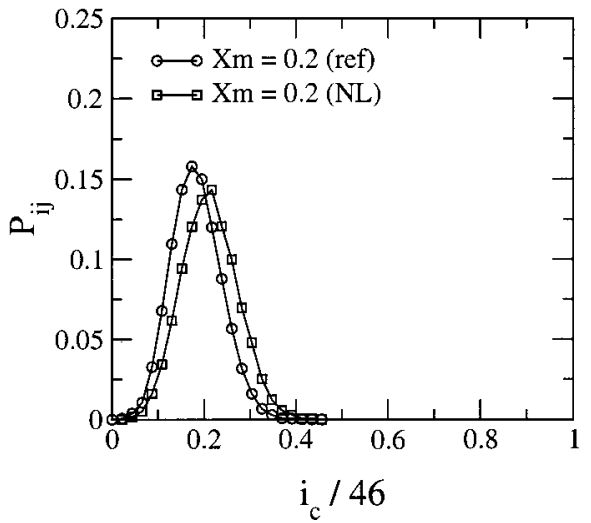

FIG. 5. The normalized probability distribution versus the monomer mole fraction in the clusters containing 46 molecules, $x_{m}^{c}=i_{c} / 46$. Here $i_{c}$ is the monomer number in the clusters. The results are obtained at total molecule number 4000 and volume $200000 \sigma^{3}$ for (a) the monomer-rich vapor $x_{m}$ $=0.8$ and $(\mathrm{b})$ the dimer-rich vapor $x_{m}=0.2$.

$p_{\mathrm{A}}$ and $j$ monomers of $B$ molecules from the system at pressure $p_{\mathrm{B}}$ and forming an $i j$-cluster at the pressure $p$. Note that this interpretation is a straightforward extension of the definition of $\Delta G_{i}$ for unary systems, ${ }^{24}$ which can be easily extended to multicomponent systems.

Using a Monte Carlo simulation method, we have computed the equilibrium cluster distribution in supersaturated vapors for both a partially miscible system and a reference system with ideal mixing behavior. The mutual enhancement of homogeneous vapor nucleation in the partially miscible system compared to the reference system is seen in the monomer-rich vapor, in agreement with the theoretical prediction of Napari and Laaksonen. The simulation confirms the physical explanation that the mutual enhancement of nucleation is due to the orientational surface order of dimers at the surface of clusters, which leads to a reduction of vapor-liquid surface tension. Presumably, this physical explanation is applicable to the real water/higher alcohol binary nucleation. Our simulation also demonstrates that the mutual enhancement of nucleation can also occur in dimer-rich vapors when the vapors are highly supersaturated.

\section{ACKNOWLEDGMENT}

One of the authors (X.C.Z.) thanks the National Science Foundations for support of this work.

\section{APPENDIX: LAW OF MASS ACTION}

A supersaturated binary vapor at metastable or constrained equilibrium with a given pressure $p$, temperature $T$, and mole fraction $x_{\mathrm{A}}$ entails the following chemical reactions (i.e., formation of $i j$-clusters):

$$
i \mathrm{~A}+j \mathrm{~B} \rightleftharpoons \mathrm{A}_{i} \mathrm{~B}_{j} .
$$

The Gibbs free energy of the system can be given by

$$
G(p)=\sum_{i, j} n_{i j} \mu_{i j}\left(p_{i j}\right)
$$

where the chemical potential of the $i j$-cluster has a $p_{i j}$ (the partial pressure of $i j$-clusters) dependence at equilibrium. Now, we consider an infinitesimal process of converting $i d \xi$ molecules of $\mathrm{A}$ and $j d \xi$ molecules of $\mathrm{B}$ into $d \xi$ number of $\mathrm{A}_{i} \mathrm{~B}_{j}$ clusters. Since the process is infinitesimal, none of the intensive properties of the system will change and thus, all the $\mu$ 's are constant during the process. The change in $G$ is therefore

$$
\begin{aligned}
d G(p) & =\mu_{\mathrm{A}}\left(p_{\mathrm{A}}\right) d n_{\mathrm{A}}+\mu_{\mathrm{B}}\left(p_{\mathrm{B}}\right) d n_{\mathrm{B}}+\mu_{i j}\left(p_{i j}\right) d n_{i j}, \\
& =\left(-i \mu_{\mathrm{A}}\left(p_{\mathrm{A}}\right)-j \mu_{\mathrm{B}}\left(p_{\mathrm{B}}\right)+\mu_{i j}\left(p_{i j}\right)\right) d \xi=\Delta \mu d \xi
\end{aligned}
$$

Since the system itself is at equilibrium, hence, according to the second law, $d G=0$. Thus, $\Delta \mu=0$ because $d \xi$ is arbitrary, or

$$
\mu_{i j}\left(p_{i j}\right)=i \mu_{\mathrm{A}}\left(p_{\mathrm{A}}\right)+j \mu_{\mathrm{B}}\left(p_{\mathrm{B}}\right),
$$

which is the law of mass action.

${ }^{1}$ A. Laaksonen, V. Talanquer, and D. W. Oxtoby, Annu. Rev. Phys. Chem. 46, 489 (1995).

${ }^{2}$ H. Reiss, J. Chem. Phys. 18, 840 (1950).

${ }^{3}$ G. J. Doyle, J. Chem. Phys. 35, 795 (1961).

${ }^{4}$ G. Wilemski, J. Chem. Phys. 80, 1370 (1984); J. Phys. Chem. 91, 2492 (1987).

${ }^{5}$ G. Wilemski, J. Chem. Phys. 62, 3763 (1975).

${ }^{6}$ J. L. Schmitt, J. Whitten, G. W. Adams, and R. A. Zalabsky, J. Chem. Phys. 92, 3693 (1990).

${ }^{7}$ R. Strey and Y. Viisanen, J. Chem. Phys. 99, 4693 (1993).

${ }^{8}$ Y. Viisanen, R. Strey, A. Laaksonen, and M. Kulmala, J. Chem. Phys. 100, 6062 (1994).

${ }^{9}$ X. C. Zeng and D. W. Oxtoby, J. Chem. Phys. 95, 5940 (1991).

${ }^{10}$ A. Laaksonen and D. W. Oxtoby, J. Chem. Phys. 102, 5803 (1995).

${ }^{11}$ A. Laaksonen and D. W. Oxtoby, J. Chem. Phys. 106, 7268 (1997).

${ }^{12}$ V. Talanquer and D. W. Oxtoby, J. Chem. Phys. 104, 1993 (1996).

${ }^{13}$ G. K. Schenter, S. M. Kathmann, and B. C. Garrett, Phys. Rev. Lett. 82, 3484 (1999).

${ }^{14}$ C. L. Weakliem and H. Reiss, J. Chem. Phys. 99, 5374 (1993).

${ }^{15}$ K. J. Oh, X. C. Zeng, and H. Reiss, J. Chem. Phys. 107, 1242 (1997).

${ }^{16}$ B. Senger, P. Schaaf, D. S. Corti, R. Bowles, J.-C. Voegel, and H. Reiss, J. Chem. Phys. 110, 6421 (1999).

${ }^{17}$ B. Senger, P. Schaaf, D. S. Corti, R. Bowles, D. Pointu, J.-C. Voegel, and H. Reiss, J. Chem. Phys. 110, 6438 (1999).

${ }^{18}$ I. Kusaka, Z.-G. Wang, and J. H. Seinfeld, J. Chem. Phys. 108, 3416 (1998).

${ }^{19}$ I. Kusaka and D. W. Oxtoby, J. Chem. Phys. 110, 5249 (1999).

${ }^{20}$ P. R. ten Wolde and D. Frenkel, J. Chem. Phys. 109, 9901 (1998).

${ }^{21}$ G. T. Gao, K. J. Oh, and X. C. Zeng, J. Chem. Phys. 110, 2533 (1999).

${ }^{22}$ K. Yasuoka and M. Matsumoto, J. Chem. Phys. 109, 8451 (1998).

${ }^{23}$ K. Yasuoka and M. Matsumoto, J. Chem. Phys. 109, 8463 (1998).

${ }^{24}$ K. J. Oh and X. C. Zeng, J. Chem. Phys. 110, 4471 (1999).

${ }^{25}$ K. J. Oh and X. C. Zeng, J. Chem. Phys. 112, 294 (2000). 
${ }^{26}$ H. Reiss and R. K. Bowles, J. Chem. Phys. 111, 7501 (1999).

${ }^{27}$ B. N. Hale and S. M. Kathmann, in Nucleation and Atmospoheric Aerosols 1996, edited by M. Kulmala and P. E. Wagner (Elsevier Science, Oxford, 1996).

${ }^{28}$ I. Kusaka, Z. G. Wang, and J. H. Seinfeld, J. Chem. Phys. 108, 6829 (1998).

${ }^{29}$ P. R. ten Wolde and D. Frenkel, J. Chem. Phys. 109, 9919 (1998).
${ }^{30}$ H. Vehkamäki, P. Paatero, M. Kulmala, and A. Laaksonen, J. Chem. Phys. 101, 9997 (1994).

${ }^{31}$ R. Strey, Y. Viisanen, and P. E. Wagner, J. Chem. Phys. 103, 4333 (1995).

${ }^{32}$ V. Talanquer and D. W. Oxtoby, J. Chem. Phys. 106, 3673 (1997).

${ }^{33}$ I. Napari and A. Laaksonen, J. Chem. Phys. 111, 5485 (1999).

${ }^{34}$ I. Napari and A. Laaksonen, Phys. Rev. Lett. 84, 2184 (2000).

${ }^{35}$ J. A. Barker and R. O. Watts, Chem. Phys. Lett. 3, 144 (1969).

${ }^{36}$ F. J. Stillinger, J. Chem. Phys. 38, 1486 (1963). 